\title{
Association of frontal gray matter volume and cerebral perfusion in heroin addiction: a multimodal neuroimaging study
}

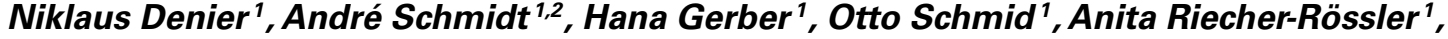 \\ Gerhard A. Wiesbeck ${ }^{1}$, Christian G. Huber ${ }^{1}$, Undine E. Lang ${ }^{1}$, Ernst-Wilhelm Radue ${ }^{1,2}$, Marc Walter ${ }^{1}$ and \\ Stefan Borgwardt ${ }^{1,2 *}$
}

1 Department of Psychiatry (UPK), University of Basel, Basel, Switzerland

${ }^{2}$ Medical Image Analysis Centre, University Hospital Basel, Basel, Switzerland

Edited by:

Qiyong Gong, West China Hospital of Sichuan University, China

Reviewed by:

Rex Eugene Jung, University of New Mexico, USA

Jiansong Xu, Yale University, USA

*Correspondence:

Stefan Borgwardt, Department of Psychiatry, University of Basel,

Wilhelm Klein-Strasse 27, CH-4012

Basel, Switzerland

e-mail: stefan.borgwardt@upkbs.ch
Structure and function are closely related in the healthy human brain. In patients with chronic heroin exposure, brain imaging studies have identified long-lasting changes in gray matter (GM) volume. More recently, we showed that acute application of heroin in dependent patients results in hypoperfusion of fronto-temporal areas compared with the placebo condition. However, the relationship between structural and cerebral blood flow (CBF) changes in heroin addiction has not yet been investigated. Moreover, it is not known whether there is any interaction between the chronic structural changes and the short and long-term effects on perfusion caused by heroin. Using a double-blind, within-subject design, heroin or placebo (saline) was administered to 14 heroin-dependent patients from a stable heroin-assisted treatment program, in order to observe acute short-term effects. Arterial spin labeling (ASL) was used to calculate perfusion quantification maps in both treatment conditions, while Voxel-Based Morphometry (VBM) was conducted to calculate regional GM density. VBM and ASL data were used to calculate homologous correlation fields by Biological Parametric Mapping (BPM) and a whole-brain Pearson $r$ correlation. We correlated each perfusion condition (heroin and placebo) separately with a VBM sample that was identical for the two treatment conditions. It was assumed that heroin-associated perfusion is manifested in short-term effects, while placebo-associated perfusion is more related to long-term effects. In order to restrict our analyses to fronto-temporal regions, we used an explicit mask for our analyses. Correlation analyses revealed a significant positive correlation in frontal areas between GM and both perfusion conditions (heroin and placebo). Heroin-associated perfusion was also negatively correlated with GM in the inferior temporal gyrus on both hemispheres. These findings indicate that, in heroin-dependent patients, low GM volume is positively associated with low perfusion within frontal regions.

Keywords: heroin addiction, biological parametric mapping, arterial spin labeling, voxel-based morphometry

\section{INTRODUCTION}

Heroin dependence is associated with chronic exposure to heroin and is characterized by compulsive drug use despite negative consequences, including social drift (downward social mobility) (1). Cognitive processes, including impulse control (control of wishes and urges), are impaired after acute and chronic heroin exposure, as indicated by behavioral studies (2). Impaired impulse control in heroin addiction is reflected by imaging activity patterns that resemble those of immature brains $(3,4)$. These functionally abnormal activities with impulsive decision-making may be related to a relapse of heroin addiction. The compulsion to use heroin is frequently driven by enhanced stress responses and craving (desire for consumption) $(5,6)$, while acute heroin administration contributes to a reduction in these negative emotions, as indicated by reduced anxiety, craving, and stress hormone release (7-9). When heroin is administered, it crosses the blood brain barrier very rapidly in comparison to other opioids (10). Within the brain, the heroin metabolites 6-monoacetylmorphine (6-MAM) and morphine bind as agonists to $\mu$ and $\kappa$ receptors (11). Both receptor types are found over the whole-brain $(12,13)$.

Structural magnetic resonance imaging (MRI) studies on the long-term effect of opioid application have demonstrated that opioid dependent subjects showed decreased gray matter (GM) in the bilateral prefrontal cortex and bilateral temporal cortex, including the insula (14). Furthermore, Yuan et al. (15) found that the GM of prefrontal, temporal, and insular regions was negatively correlated with the duration of heroin use, suggesting that heroin consumption has a cumulative effect.

Studies on cerebral blood flow (CBF) in heroin addiction have showed that chronic heroin use is associated with a decrease in global perfusion, with the greatest decrease in the frontal, occipital, and temporal regions (16). In our recently published study, we observed acute effects of heroin on cerebral perfusion (17). Our findings indicate that, in comparison to placebo, heroin leads to 
hypoperfusion in the left anterior cingulate cortex (ACC), the left medial prefrontal cortex ( $\mathrm{mPFC}$ ), and in bilateral insula.

However, the relationship between GM volume and perfusion in heroin addiction has not yet been investigated. In the current study, we examined whether there was an association between GM volume and perfusion in 15 heroin-addicted patients. Perfusion was assessed during the normal drug free condition (placebo) and after administration of heroin. We applied Biological Parametric Mapping (BPM) to assess the relation between perfusion - as measured by arterial spin labeling (ASL) - and GM volume as measured by Voxel-Based Morphometry (VBM). The BPM toolbox was developed by Casanova and colleagues in MATLAB (18). This incorporates information obtained from other modalities as regressors for whole-brain analyses and also allows voxel-wise multimodal correlation. BPM has been used widely for integrative analysis of different neuroimaging modalities (19-21). On the basis of our previous findings (17), we expected a direct relationship between perfusion and GM within the areas with the most marked hypoperfusion during the acute effects of heroin. We hypothesized that, within the medial frontal and the temporal cortex, low perfusion should correlates with low GM volume.

\section{MATERIALS AND METHODS STUDY SAMPLE}

The study was approved by the local ethics committee and registered under http://clinicaltrials.gov (ID NCT01174927). After receiving a written and oral description of the study aims, all participants gave their written informed consent before inclusion.

Fourteen (eight male, six female; mean age $40.7 \pm 6.8$ years) non-left-handed heroin-dependent out-patients were recruited from the Center of Substance Use Disorders of the Department of Psychiatry in Basel University. The inclusion criteria were as follows: age older than 18 years, current heroin-maintained treatment for at least 6 months, with an unchanged heroin dose during the past 3 months. The exclusion criteria were a positive alcohol breathalyzer test, or an additional physical disease or psychiatric disorder other than substance dependencies. Clinically experienced psychiatrists conducted the Structured Clinical Interview for DSM-IV Axis II Disorders (SCID-II) (22) to assess the diagnosis of comorbid personality disorders.

Subjects reported their age of first heroin use (mean $=18.4 \pm$ 2.9 years), years of heroin dependence ( mean $=21.4 \pm 7.2$ years), duration of heroin maintenance (mean $=7.2 \pm 3.9$ years), and daily heroin dose $($ mean $=352 \pm 178 \mathrm{mg}$ ). Patients were told to abstain from illicit drug use other than the prescribed heroin for the duration of the study, from alcohol intake for $72 \mathrm{~h}$ and from tobacco consumption for $2 \mathrm{~h}$ before scanning. Illicit substances and medications were controlled by a urine test at each session. Nevertheless, three patients were tested positive for cannabis and six patients for cocaine at one or both points of the measurement.

\section{DRUG ADMINISTRATION}

In a cross-over, double-blind design, placebo ( $5 \mathrm{ml}$ saline), and half of the daily heroin dose (mean $=176 \pm 89.1 \mathrm{mg}$ ) were administered intravenously over a period of $30 \mathrm{~s}$ by a study nurse $20 \mathrm{~min}$ before the scanning session started. Heroin was dissolved in $5 \mathrm{ml}$ of sterile water and aspirated into a syringe, according to the procedure described by Stohler et al. (23). Each patient was scanned twice, with a short interval between scans (mean $8.4 \pm 3.3$ days). On 1 day, patients received an injection of heroin or placebo (saline) before the scan and on the other day after the scan. Patients received their regular morning dose of heroin, corresponding to half of their daily individual dose.

\section{IMAGE ACQUISITION}

Scanning was performed on a 3T MRI scanner (Magnetom Verio, Siemens Healthcare, Germany), using a 3D T1-weighted sequence (MPRAGE) for high resolution anatomical data and an ASL sequence (24) for quantification of CBF (perfusion). MPRAGE parameters were $1 \mathrm{~mm} \times 1 \mathrm{~mm} \times 1 \mathrm{~mm}$ isotropic resolution, repetition time of $2000 \mathrm{~ms}$, inversion time of $1000 \mathrm{~ms}$, and echo time of $3.4 \mathrm{~ms}$. ASL was based on a flow-sensitive alternating inversion recovery spin labeling scheme (25), combined with modified Q2TIPS (TI periodic saturation) pulse preparation and a single-shot 3D gradient-spin echo readout (26). The sequence parameters were: repetition time $3200 \mathrm{~ms}$, echo time $12.7 \mathrm{~ms}$, and spatial resolution $4.6 \mathrm{~mm} \times 4.6 \mathrm{~mm} \times 4 \mathrm{~mm}$ (interpolated to $2.3 \mathrm{~mm} \times 2.3 \mathrm{~mm} \times 4 \mathrm{~mm}$ ). Further details of ASL acquisition have been described elsewhere (17).

\section{PREPROCESSING OF PERFUSION DATA}

Cerebral blood flow (perfusion maps) was calculated by in-house software from ASL DICOM data. The difference images of the label and control images were first calculated and the time course was fitted to an ideal flow model. The equation for perfusion was then solved (24). The resulting perfusion maps were expressed in the unit $\mathrm{ml} / 100 \mathrm{~g} / \mathrm{min}$. Conversion from the Metafile (MHD) to the NIFTI format was performed with MedINRIA Software ${ }^{1}$. Further preprocessing was conducted by Statistical Parametric Mapping $\left(\mathrm{SPM}^{2}\right)$, running under the MATLAB environment. Perfusion maps were first realigned and then masked with binarized intracranial tissue (binarization threshold: voxel intensity $>0.1$ ) to remove the extracerebral signal. Perfusion maps were then normalized to MNI space and smoothed using a $6 \mathrm{~mm}$ full-width-at halfmaximum (FWHM) Gaussian kernel and proportionally scaled. More details of perfusion map preprocessing have been described elsewhere (17).

Voxel-Based Morphometry Structural data (MPRAGE) were preprocessed with VBM implemented in SPM8. MPRAGE images were non-rigidly normalized to a population-based average using diffeomorphic anatomical registration through exponentiated lie algebra (DARTEL) (27). The segmented tissue maps of GM were modulated with the Jacobian determinants from the spatial normalization to correct for volume changes. Finally, images were smoothed with an $8 \mathrm{~mm}$ FWHM Gaussian kernel.

Between condition differences were assessed by a voxel-wise whole-brain analysis using a general linear model (GLM). Statistical significance was assessed at cluster-level, using non-stationary random field theory (28). The first step of this cluster-level inference strategy consisted of identifying spatially contiguous

\footnotetext{
${ }^{1}$ http://www-sop.inria.fr/asclepios/software/MedINRIA/

${ }^{2}$ http://www.fil.ion.ucl.ac.uk/spm/
} 


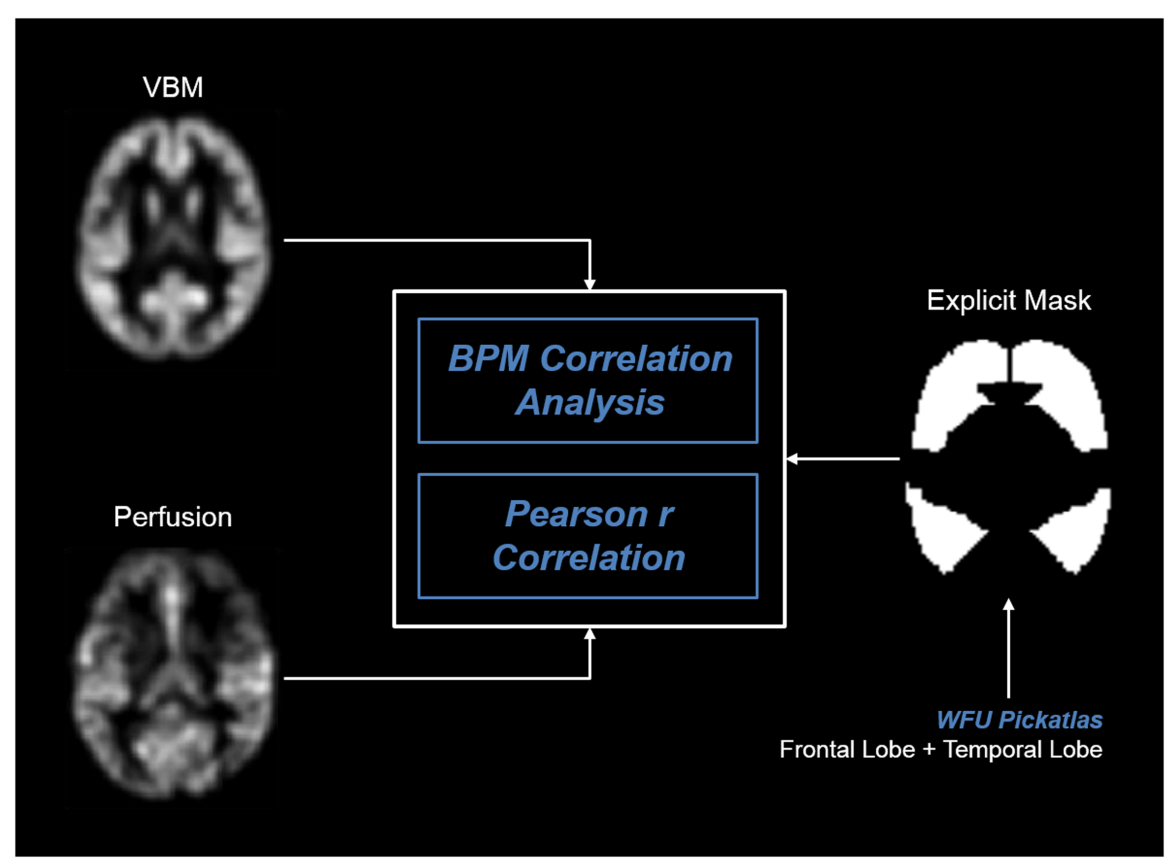

FIGURE 1 | BPM analysis pipeline of correlation analyses.

Table 1 | Correlation between gray matter with placebo-associated perfusion.

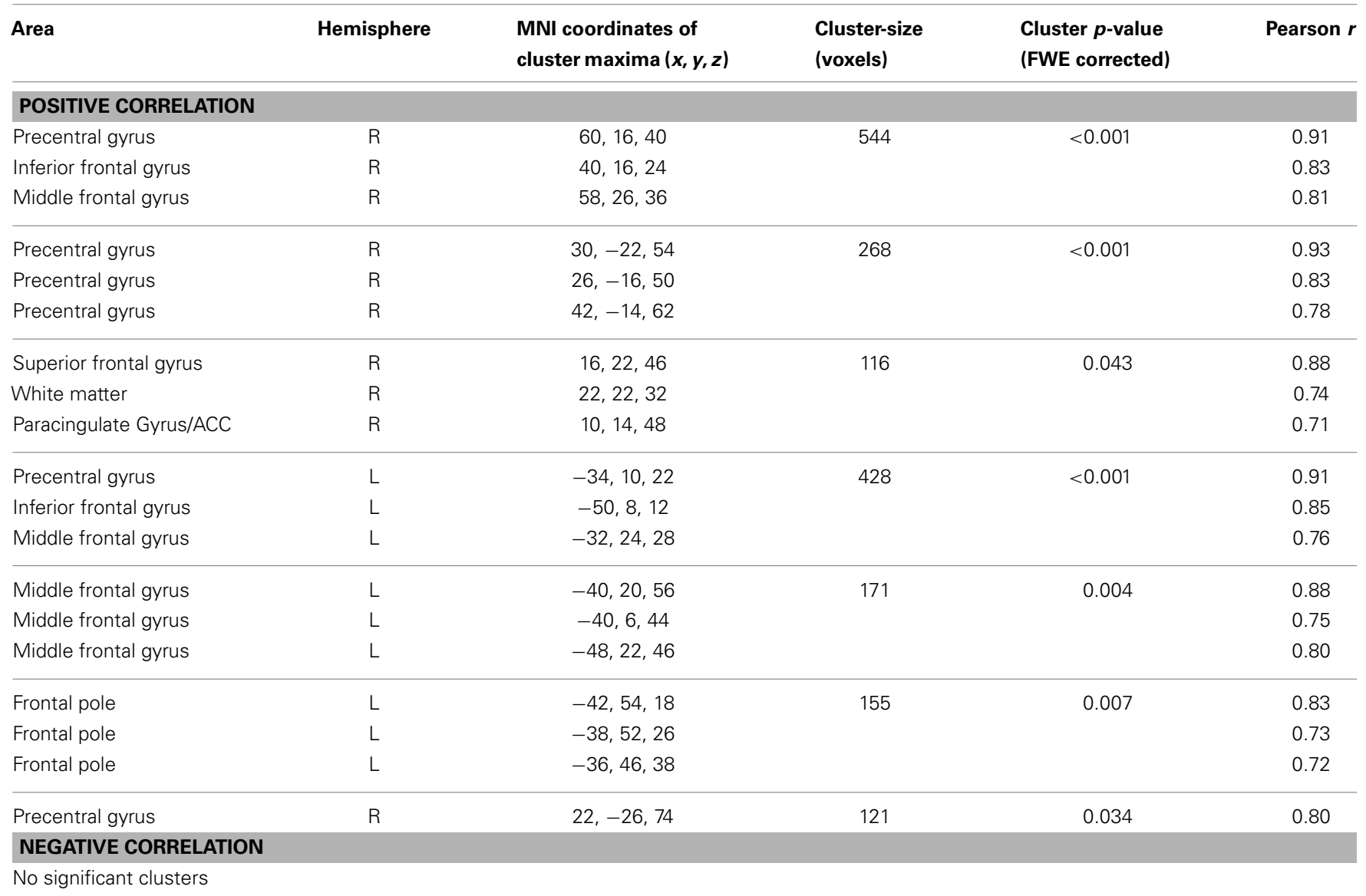

$L$, left; $R$, right. 
voxels at a threshold of $p<0.01$, without correction (clusterforming threshold) (29). Finally, a family-wise error (FWE) corrected cluster-extent threshold of $p<0.05$ was defined in order to infer statistical significance. A paired $t$ test was used to examine the following contrasts: $\mathrm{GM}_{\text {heroin }}<\mathrm{GM}_{\text {placebo }}$ and $\mathrm{GM}_{\text {heroin }}>\mathrm{GM}_{\text {placebo }}$.

\section{BIOLOGICAL PARAMETRIC MAPPING}

For an integrative analysis of multimodal imaging data, we used $\mathrm{BPM}^{3}$ (18) running with SPM5 software. Preprocessed perfusion and VBM maps were realigned and used for whole-brain voxelto-voxel correlation analysis. In order to restrict our analyses to fronto-temporal regions, that we had found to be hypoperfused during heroin (17), we used an explicit mask of the frontal and temporal lobe by WFU PickAtlas ${ }^{4}$. For overview, we refer to Figure 1.

In BPM, each perfusion condition (heroin and placebo) was correlated separately with the VBM data. Cluster-level inference was performed using a homologous correlation field (30) and a cluster-forming threshold of $p<0.01$. Significant clusters were corrected for multiple comparisons with a FWE of $p<0.05$ (29). MNI coordinates of significant clusters were converted into Talairach space and labeled with the Talairach Client 2.4.35 .

In addition, we calculated Pearson $r$ correlations between perfusion and VBM data for each voxel within the standardized MNI space. We used the image calculation function implemented in SPM8 and applied the Pearson formula $r=\frac{\Sigma_{i}^{14}\left(x_{i}-\bar{x}\right)\left(y_{i}-\bar{y}\right)}{\sqrt{\Sigma_{i}^{14}\left(x_{i}-\bar{x}\right) \cdot \Sigma_{i}^{14} y_{i}-\bar{y}}}$ to our preprocessed images, where $x_{\mathrm{i}}$ and $y_{\mathrm{i}}$ represent perfusion and VBM values, and $\bar{x}$ and $\bar{y}$ the mean perfusion and mean VBM values over the patient's group, respectively $(n=14)$.

\section{RESULTS}

\section{VOXEL-BASED MORPHOMETRY ANALYSES}

Comparison of the heroin and placebo conditions found no significant difference in either direction (heroin $>$ placebo and heroin $<$ placebo).

\section{MULTIMODAL CORRELATION ANALYSES}

Biological Parametric Mapping analysis integrating GM and perfusion data after the placebo treatment revealed a significant positive correlation between perfusion and GM volume in frontal areas on both hemispheres including the precentral gyrus, the inferior, middle and superior frontal gyrus, the frontal pole, and the right paracingulate cortex, including parts of the ACC (Table 1 and Figure 2, upper panel). Pearson $r$ values within the cluster maxima were all $r>0.7$. No significant cluster was found in the temporal lobe and there was no significant negative correlation between modalities (Table 1).

Biological Parametric Mapping analysis integrating GM and perfusion data after the heroin treatment also showed a positive correlation between perfusion and GM volume in frontal areas. Significant clusters were found in the left precentral gyrus, the left

${ }^{3}$ http://www.nitrc.org/projects/rbpm/

${ }^{4}$ http://fmri.wfubmc.edu/software/pickatlas

${ }^{5} \mathrm{http} / / /$ www.talairach.org/client.html

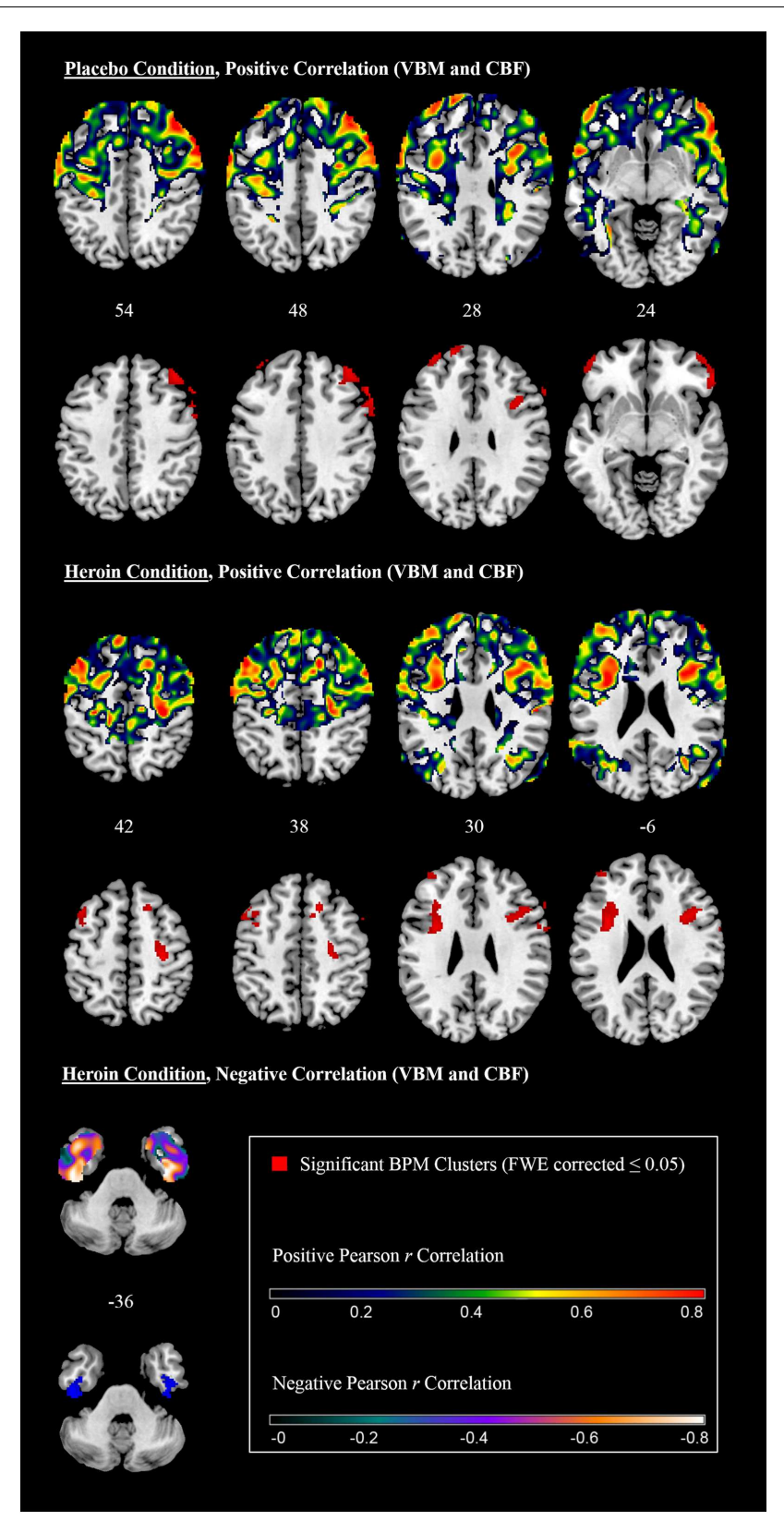

FIGURE 2 | Correlation between gray matter volume and perfusion (axial slices).

middle and inferior frontal gyrus and the frontal pole on both hemispheres (Table 2 and Figure 2, middle panel). No significant cluster was found in the temporal lobe. Pearson $r$ values within the cluster maxima were all $r>0.7$. A negative correlation was found in the inferior temporal gyrus and the temporal fusiform cortex on both hemispheres (Table 2 and Figure 2, lower panel). Pearson $r$ values within the cluster maxima were all less than -0.7 .

\section{DISCUSSION}

In the present study, we examined the relationship between measurements of two different neuroimaging modalities in 
Table 2 | Correlation between gray matter with heroin-associated perfusion.

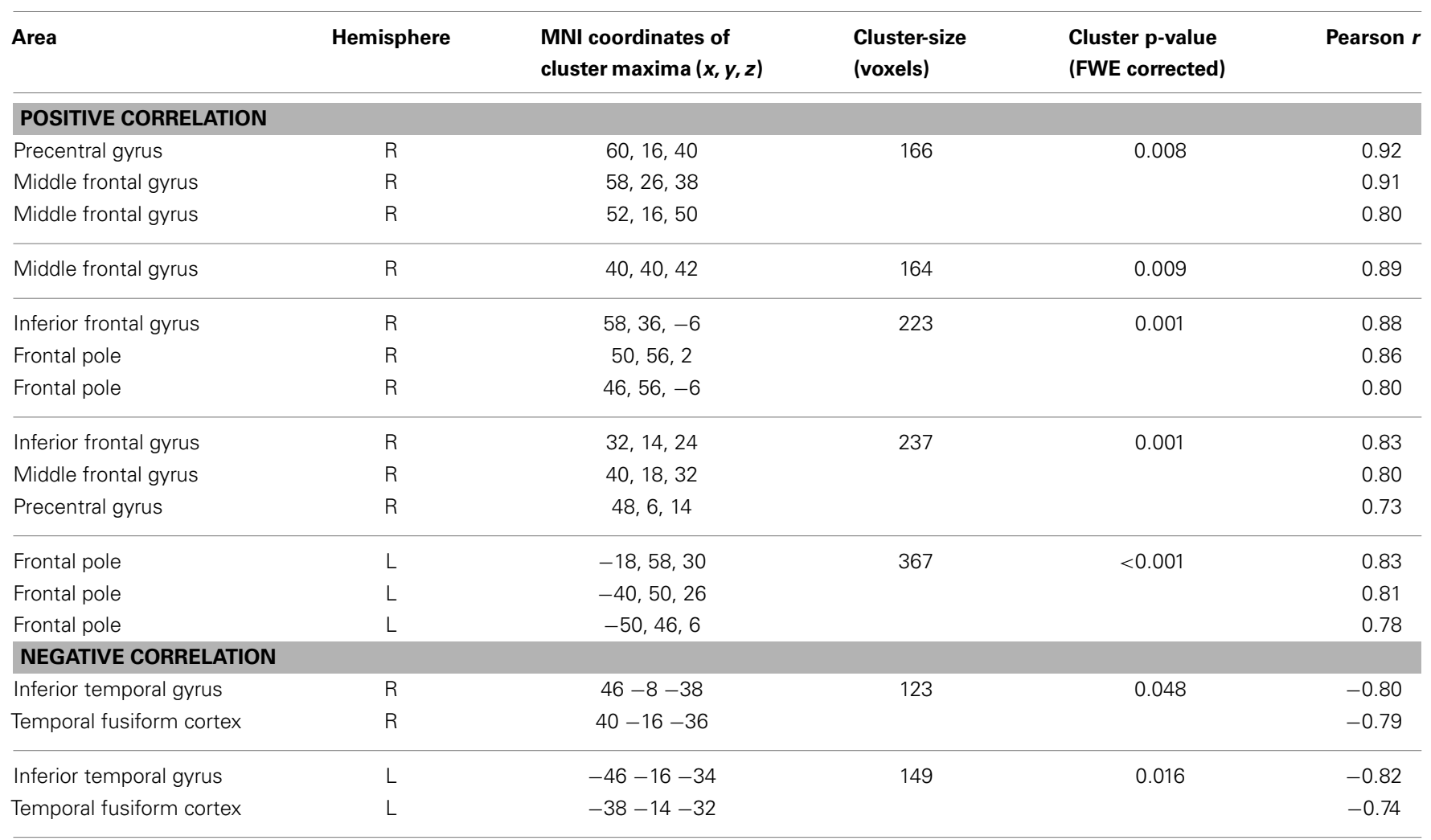

$L$, left; $R$, right.

heroin-dependent patients, in particular between GM volumes and perfusion. We compared perfusion maps during acute heroin treatment and during placebo treatment with GM volumes by performing BPM correlations. We found that both placebo and heroin perfusion correlated positively with GM in frontal brain regions. Perfusion during placebo treatment, which we assumed to be related to the long-term effects of heroin, was also positively associated with GM in the cortical midline structure (ACC). We also found that heroin-associated perfusion correlated negatively with GM in the inferior temporal gyrus on both hemispheres. This negative correlation is difficult to explain in terms of our hypothesis that hypoperfusion is the driving force leading to GM reduction. The cuneus serves as visual processing and inhibitory control centers and electroencephalographic studies have revealed abnormalities in heroin-dependent individuals $(31,32)$ and decreased regional homogeneity (33) in this region. In our VBM analysis we found, as expected, no significant difference between heroin and placebo condition.

The regions showing a positive correlation between perfusion and GM are in line with regions showing reduced GM in heroin dependence $(14,15,34,35)$. Single photon emission computed tomography studies have also shown decreased perfusion in frontal and temporal cortex in opioid dependent patients (16, 36-38).

The frontal cortex is known to play an essential role in heroin addiction. In a recent study, we could show that heroin impairs stimulus-driven attention allocation, as indicated by reduced activity in the right inferior frontal gyrus (39). Further studies showed that loss of control over drug intake is not only a result of disrupted subcortical reward circuits but also of prefrontal higher-order executive functions (40). A recent study showed that prefrontal impairments in heroin addiction are directly associated with increases in impulsivity and the duration of heroin dependence (41). Studies with resting-state functional MRI in heroin addicts showed a decreased amplitude of low-frequency fluctuations in fronto-temporal regions (42), reduced regional homogeneity in the bilateral medial orbitofrontal cortex (33), and decreased functional connectivity in right dorsal ACC (43). Moreover, graph theoretical analysis demonstrated abnormal topological properties in areas of drug addiction-related circuits (44). Heroin addicts also showed altered default mode and rostral ACC network properties (45).

However, the question of how perfusion and GM interact in heroin dependence was not answered in our analysis. A possible explanation could be that heroin-associated perfusion in prefrontal and extended limbic areas may alter cerebral volume due to mild but chronic hypoxia and ischemia. This hypothesis is in line with animal models of brain ischemia, which showed that reduction in perfusion leads to neural death and cognitive impairment $(46,47)$. It is known that a heroin overdose can lead to coma with depressed respiration and increased pulse rate (48). These comas could cause a variety of neurological complications, including post-anoxic encephalopathy and cerebrovascular infarctions $(49,50)$. Interestingly, a study showed that GM differences between heroin addicts and a control group diminish 
after only 1 month heroin abstinence (51). This also supports the idea that GM deficits are caused by heroin itself and are not pre-existing before heroin addiction. The hypothesis that hypoperfusion leads to GM volume reduction by metabolic impairment, affecting neural, and glial function, is also in line with studies showing impaired white matter integrity in long-term addiction (52, 53). White matter microstructure deficits in heroin users are also associated with the duration of heroin dependence and impaired decision-making (54).

There are some limitations to this study, which should be carefully considered in interpreting our findings. Firstly, we only used a moderate number of subjects. A second limitation is the lack of a control group. It was thus not possible to analyze regional GM and perfusion differences with a between group approach and it therefore remains unclear how placebo-associated perfusion, reflecting normal perfusion in heroin-dependent patients, differs from that in healthy controls. A further difficulty is the aim of separating pure heroin effects on perfusion and structure

\section{REFERENCES}

1. Leshner AI. Addiction is a brain disease, and it matters. Science (1997) 278:45-7. doi:10.1126/science.278. 5335.45

2. Koob GF, Le Moal M. Drug abuse: hedonic homeostatic dysregulation. Science (1997) 278:52-8. doi:10. 1126/science.278.5335.52

3. Lee TM,Zhou WH, Luo XJ, Yuen KS, Ruan XZ, Weng XC. Neural activity associated with cognitive regulation in heroin users: a fMRI study. $\mathrm{Neu}$ rosci Lett (2005) 382:211-6. doi:10. 1016/j.neulet.2005.03.053

4. Lin B, Qian RB, Fu XM, Ji XB, Wei XP, Niu CS, et al. Impulsive decision-making behaviors in heroin addicts: a study of functional magnetic resonance imaging. Zhonghua Yi Xue Za Zhi (2012) 92:1033-6.

5. Le Moal M, Koob GF. Drug addiction: pathways to the disease and pathophysiological perspectives. Eur Neuropsychopharmacol (2007) 17:377-93. doi:10.1016/j. euroneuro.2006.10.006

6. Thayer RE, Hutchison KE. Neuroimaging in clinical studies of craving: importance of reward and control networks. Psychol Addict Behav (2013) 27:543-6. doi:10. 1037/a0030275

7. Gerber H, Borgwardt SJ, Schmid O, Gerhard U, Joechle W, Riecher-Rossler A, et al. The impact of diacetylmorphine on hypothalamic-pituitary-adrenal axis activity and heroin craving in heroin dependence. Eur Addict Res (2012) 18:116-23. doi:10.1159/000334411

8. Blum J, Gerber H, Gerhard U, Schmid O, Petitjean S,
Riecher-Rössler A, et al. Acute effects of heroin on emotions in heroin-dependent patients. Am J Addict (2013). doi:10.1111/j.15210391.2013.12025.x

9. Walter M, Gerber H, Kuhl HC, Schmid O, Joechle W, Lanz C, et al. Acute effects of intravenous heroin on the hypothalamicpituitary-adrenal axis response: a controlled trial. J Clin Psychopharmacol (2013) 33:193-8. doi:10. 1097/JCP.0b013e31828393cb

10. Rook EJ, Huitema AD, Van Den Brink W, Van Ree JM, Beijnen JH. Pharmacokinetics and pharmacokinetic variability of heroin and its metabolites: review of the literature. Curr Clin Pharmacol (2006) 1:109-18. doi:10.2174/ 157488406775268219

11. Boix F, Andersen JM, Morland J. Pharmacokinetic modeling of subcutaneous heroin and its metabolites in blood and brain of mice. Addict Biol (2013) 18(1):1-7. doi: 10.1111/j.1369-1600.2010.00298.x

12. Pfeiffer A, Pasi A, Mehraein P, Herz A. Opiate receptor binding sites in human brain. Brain Res (1982) 248:87-96. doi:10.1016/ 0006-8993(82)91150-7

13. Maurer R, Cortes R, Probst A, Palacios JM. Multiple opiate receptor in human brain: an autoradiographic investigation. Life Sci (1983) 33(Suppl 1):231-4. doi:10. 1016/0024-3205(83)90485-X

14. Lyoo IK, Pollack MH, Silveri MM, Ahn KH, Diaz CI, Hwang J, et al. Prefrontal and temporal gray matter density decreases in opiate dependence. Psychopharmacology (Berl) (2006) 184:139-44. doi: $10.1007 / \mathrm{s} 00213-005-0198-\mathrm{x}$

from effects of other substances. Abstinence from tobacco use may induce craving, resulting in increased perfusion in areas associated with cigarette craving (55). However, all patients were smokers and therefore nicotine may be ruled out as confounding factor. In our previous analysis, we concluded that cocaine and cannabis did not significantly modify perfusion in our patient group (17).

In conclusion, we demonstrated that local GM volumes at baseline in heroin-dependent patients predicted perfusion in frontal regions after administration of placebo and heroin. These findings may suggest that recurrent and chronic hypoperfusion induced by heroin is a driving force for reduced GM in heroin addiction.

\section{ACKNOWLEDGMENTS}

All authors have agreed to the submission of this article in this form, and we do not have any conflict of interests that might be interpreted as influencing its content. The authors reported no biomedical financial interests or potential conflicts of interest.

15. Yuan Y, Zhu Z, Shi J, Zou Z, Yuan F, Liu Y, et al. Gray matter density negatively correlates with duration of heroin use in young lifetime heroin-dependent individuals. Brain $\operatorname{Cog} n$ (2009) 71:223-8. doi:10.1016/j.bandc.2009.08.014

16. Botelho MF, Relvas JS, Abrantes M, Cunha MJ, Marques TR, Rovira E, et al. Brain blood flow SPET imaging in heroin abusers. Ann N Y Acad Sci (2006) 1074:466-77. doi:10.1196/ annals.1369.047

17. Denier N, Gerber $H$, Vogel $M$, Klarhofer M, Riecher-Rossler A, Wiesbeck GA, et al. Reduction in cerebral perfusion after heroin administration: a resting state arterial spin labeling study. PLoS ONE (2013) 8:e71461. doi:10.1371/ journal.pone.0071461

18. Casanova R, Srikanth R, Baer A, Laurienti PJ, Burdette JH, Hayasaka S, et al. Biological parametric mapping: a statistical toolbox for multimodality brain image analysis. Neuroimage (2007) 34:137-43. doi:10. 1016/j.neuroimage.2006.09.011

19. Fusar-Poli P, McGuire P, Borgwardt S. Mapping prodromal psychosis: a critical review of neuroimaging studies. Eur Psychiatry (2012) 27(3):181-91. doi:10.1016/j.eurpsy. 2011.06 .006

20. Smieskova R, Allen P, Simon A, Aston J, Bendfeldt K, Drewe J, et al. Different duration of at-risk mental state associated with neurofunctional abnormalities. a multimodal imaging study. Hum Brain Mapp (2012) 33(10):2281-94. doi: $10.1002 / \mathrm{hbm} .21360$

21. Bustamante JC, Barrós-Loscertales A, Ventura-Campos N, Sanjuán A, Llopis JJ, Parcet MA, et al.
Right parietal hypoactivation in a cocaine-dependent group during a verbal working memory task. Brain Res (2011) 1375: 111-9. doi:10.1016/j.brainres.2010. 12.042

22. First M, Gibbon M, Spitzer R, Williams J, Benjamin L. Structured Clinical Interview for DSM-IV Axis II Personality Disorders, (SCID-II). New York: American Psychiatric Press, Inc (1997).

23. Stohler R, Dursteler KM, Stormer R, Seifritz E, Hug I, Sattler-Mayr J, et al. Rapid cortical hemoglobin deoxygenation after heroin and methadone injection in humans: a preliminary report. Drug Alcohol Depend (1999) 57:23-8. doi:10. 1016/S0376-8716(99)00036-8

24. Gunther M, Oshio K, Feinberg DA. Single-shot 3D imaging techniques improve arterial spin labeling perfusion measurements. Magn Reson Med (2005) 54:491-8. doi:10.1002/ mrm. 20580

25. Kim SG. Quantification of relative cerebral blood flow change by flow-sensitive alternating inversion recovery (FAIR) technique: application to functional mapping. Magn Reson Med (1995) 34:293-301. doi: 10.1002/mrm.1910340303

26. Amann M, Achtnichts L, Hirsch JG, Naegelin Y, Gregori J, Weier K, et al. 3D GRASE arterial spin labelling reveals an inverse correlation of cortical perfusion with the white matter lesion volume in MS. Mult Scler (2012) 18(11):1570-6. doi:10.1177/ 1352458512441984

27. Ashburner J. A fast diffeomorphic image registration algorithm. $\mathrm{Neu}$ roimage (2007) 38:95-113. doi:10. 1016/j.neuroimage.2007.07.007 
28. Hayasaka S, Phan KL, Liberzon I, Worsley KJ, Nichols TE. Nonstationary cluster-size inference with random field and permutation methods. Neuroimage (2004) 22:676-87. doi:10.1016/j. neuroimage.2004.01.041

29. Petersson KM, Nichols TE, Poline JB, Holmes AP. Statistical limitations in functional neuroimaging. II. Signal detection and statistical inference. Philos Trans $R$ Soc Lond B Biol Sci (1999) 354: 1261-81. doi:10.1098/rstb.1999. 0478

30. Cao J, Worsley KJ. The geometry of correlation fields with an application to functional connectivity of the brain. Ann Appl Prob (1999) 9:1021-57. doi:10. 1214/aoap/1029962864

31. Feinstein B. EEG findings in heroin addicts during induction and maintenance on methadone. Electroencephalogr Clin Neurophysiol (1975) 39:96-9. doi:10.1016/ 0013-4694(75)90133-9

32. Polunina AG, Davydov DM. EEG spectral power and mean frequencies in early heroin abstinence. Prog Neuropsychopharmacol Biol Psychiatry (2004) 28:73-82. doi:10.1016/ j.pnpbp.2003.09.022

33. Qiu YW, Han LJ, Lv XF, Jiang GH, Tian JZ, Zhuo FZ, et al. Regional homogeneity changes in heroindependent individuals: resting-state functional MR imaging study. Radiology (2011) 261:551-9. doi:10. 1148/radiol.11102466

34. Liu H, Hao Y, Kaneko Y, Ouyang $\mathrm{X}$, Zhang $\mathrm{Y}, \mathrm{Xu} \mathrm{L}$, et al. Frontal and cingulate gray matter volume reduction in heroin dependence: optimized voxel-based morphometry. Psychiatry Clin Neurosci (2009) 63:563-8. doi:10.1111/j.1440-1819. 2009.01989.x

35. Yuan K, Qin W, Dong M, Liu J, Sun J, Liu P, et al. Gray matter deficits and resting-state abnormalities in abstinent heroin-dependent individuals. Neurosci Lett (2010) 482:101-5. doi: 10.1016/i.neulet.2010.07.005

36. Krystal JH, Woods SW, Kosten TR, Rosen MI, Seibyl JP, Van Dyck CC, et al. Opiate dependence and withdrawal: preliminary assessment using single photon emission computerized tomography (SPECT). Am J Drug Alcohol Abuse (1995) 21:47-63. doi:10. 3109/00952999509095229

37. Rose JS, Branchey M, BuydensBranchey L, Stapleton JM, Chasten $\mathrm{K}$, Werrell A, et al. Cerebral perfusion in early and late opiate withdrawal: a technetium-99mHMPAO SPECT study. Psychiatry Res (1996) 67:39-47. doi:10.1016/ 0925-4927(96)02663-7

38. Danos P, Kasper S, Grunwald F, Klemm E, Krappel C, Broich K, et al. Pathological regional cerebral blood flow in opiate-dependent patients during withdrawal: a HMPAO-SPECT study. Neuropsychobiology (1998) 37:194-9. doi:10.1159/000026502

39. Schmidt A, Walter M, Gerber $H$, Schmid O, Smieskova R, Bendfeldt $\mathrm{K}$, et al. Inferior frontal cortex modulation with an acute dose of heroin during cognitive control. Neuropsychopharmacology (2013) 38(11):2231-9. doi:10.1038/ npp. 2013.123

40. Goldstein RZ, Volkow ND. Dysfunction of the prefrontal cortex in addiction: neuroimaging findings and clinical implications. Nat Rev Neurosci (2011) 12:652-69. doi:10. 1038/nrn3119

41. Qiu YW, Jiang GH, Su HH, Lv XF, Tian JZ, Li LM, et al. The impulsivity behavior is correlated with prefrontal cortex gray matter volume reduction in heroindependent individuals. Neurosci Lett (2013) 538:43-8. doi:10.1016/ j.neulet.2013.01.019

42. Jiang GH, Qiu YW, Zhang XL, Han LJ, Lv XF, Li LM, et al. Amplitude low-frequency oscillation abnormalities in the heroin users: a resting state fMRI study. Neuroimage (2011) 57:149-54. doi: 10.1016/j.neuroimage.2011.04.004

43. Ma N, Liu Y, Fu XM, Li N, Wang $\mathrm{CX}$, Zhang $\mathrm{H}$, et al. Abnormal brain default-mode network functional connectivity in drug addicts. PLoS ONE (2011) 6:e16560. doi:10.1371/ journal.pone.0016560

44. Yuan K, Qin W, Liu J, Guo Q, Dong $\mathrm{M}$, Sun J, et al. Altered small-world brain functional networks and duration of heroin use in male abstinent heroin-dependent individuals. Neurosci Lett (2010) 477:37-42. doi: 10.1016/j.neulet.2010.04.032

45. Yuan K, Qin W, Dong M, Liu J, Liu P, Zhang Y, et al. Combining spatial and temporal information to explore resting-state networks changes in abstinent heroin-dependent individuals. $\mathrm{Neu}$ rosci Lett (2010) 475:20-4. doi:10. 1016/j.neulet.2010.03.033

46. Otori T, Katsumata T, Muramatsu H, Kashiwagi F, Katayama Y, Terashi A. Long-term measurement of cerebral blood flow and metabolism in a rat chronic hypoperfusion model. Clin Exp Pharmacol Physio (2003) 30:266-72. doi:10.1046/j. 1440-1681.2003.03825.x

47. Cechetti F, Pagnussat AS, Worm PV, Elsner VR, Ben J, Da Costa MS, et al. Chronic brain hypoperfusion causes early glial activation and neuronal death, and subsequent long-term memory impairment. Brain Res Bull (2012) 87: 109-16. doi:10.1016/j.brainresbull. 2011.10.006

48. Richter RW, Pearson J, Bruun B, Challenor YB, Brust JC, Baden MM. Neurological complications of addiction to heroin. Bull NY Acad Med (1973) 49:3-21.

49. Protass LM. Delayed postanoxic encephalopathy after heroin use. Ann Intern Med (1971) 74:738-9. doi:10.7326/0003-4819-74-5-738

50. Brust JC, Richter RW. Stroke associated with addiction to heroin. J Neurol Neurosurg Psychiatry (1976) 39:194-9. doi:10.1136/jnnp.39.2.194

51. Wang X, Li B, Zhou X, Liao Y, Tang J, Liu $T$, et al. Changes in brain gray matter in abstinent heroin addicts. Drug Alcohol Depend (2012) 126:304-8. doi:10. 1016/j.drugalcdep.2012.05.030

52. Wang Y, Li W, Li Q, Yang W, Zhu J, Wang W. White matter impairment in heroin addicts undergoing methadone maintenance treatment and prolonged abstinence: a preliminary DTI study. Neurosci Lett (2011) 494:49-53. doi:10.1016/ j.neulet.2011.02.053
53. Shen Y, Wang E, Wang X, Lou M. Disrupted integrity of white matter in heroin-addicted subjects at different abstinent time. J Addict Med (2012) 6:172-6. doi:10.1097/ADM. 0b013e318252db94

54. Qiu Y, Jiang G, Su H, Lv X, Zhang $X$, Tian J, et al. Progressive white matter microstructure damage in male chronic heroin dependent individuals: a DTI and TBSS study. PLoS ONE (2013) 8:e63212. doi:10. 1371/journal.pone.0063212

55. Wang Z, Ray R, Faith M, Tang K, Wileyto EP, Detre JA, et al. Nicotine abstinence-induced cerebral blood flow changes by genotype. Neurosci Lett (2008) 438:275-80. doi:10. 1016/j.neulet.2008.04.084

Conflict of Interest Statement: The authors declare that the research was conducted in the absence of any commercial or financial relationships that could be construed as a potential conflict of interest.

Received: 16 August 2013; accepted: 02 October 2013; published online: 21 October 2013.

Citation: Denier N, Schmidt A, Gerber $H$, Schmid O, Riecher-Rössler $A$, Wiesbeck GA, Huber CG, Lang UE, Radue E-W, Walter $M$ and Borgwardt S (2013) Association of frontal gray matter volume and cerebral perfusion in heroin addiction: a multimodal neuroimaging study. Front. Psychiatry 4:135. doi: 10.3389/fpsyt.2013.00135

This article was submitted to Neuropsychiatric Imaging and Stimulation, a section of the journal Frontiers in Psychiatry.

Copyright (C) 2013 Denier, Schmidt, Gerber, Schmid, Riecher-Rössler, Wiesbeck, Huber, Lang, Radue, Walter and Borgwardt. This is an open-access article distributed under the terms of the Creative Commons Attribution License (CC BY). The use, distribution or reproduction in other forums is permitted, provided the original author(s) or licensor are credited and that the original publication in this journal is cited, in accordance with accepted academic practice. No use, distribution or reproduction is permitted which does not comply with these terms. 